\title{
Participation des populations aux projets programmés de migration environnementale dans le Ningxia (Chine)
}

Governance and migrant participation in environmental migration projects in Ningxia (China)

Gobernabilidad y participación de los migrantes en los proyectos de migración ambientales en Ningxia (China)

François N. Dubé, Yang HaiJuan et Huang LiJun

\section{OpenEdition}

\section{Journals}

Édition électronique

URL : https://journals.openedition.org/remi/7290

DOI : 10.4000/remi.7290

ISSN : $1777-5418$

Éditeur

Université de Poitiers

Édition imprimée

Date de publication : 1 juin 2015

Pagination : 97-118

ISBN : 979-10-90426-25-2

ISSN : 0765-0752

\section{Référence électronique}

François N. Dubé, Yang HaiJuan et Huang LiJun, « Participation des populations aux projets programmés de migration environnementale dans le Ningxia (Chine) », Revue européenne des migrations internationales [En ligne], vol. 31 - n² | 2015, mis en ligne le 01 juin 2018, consulté le 14 avril 2022. URL : http://journals.openedition.org/remi/7290; DOI : https://doi.org/10.4000/remi.7290 


\title{
Participation des populations aux projets programmés de migration environnementale dans le Ningxia (Chine)
}

\author{
François N. Dubé1, Yang HaiJuan² \\ et Huang LiJun ${ }^{3}$
}

\section{Introduction}

La relation entre changements environnementaux et déplacements de population - et particulièrement les projets de " migrations environnementales " en tant que politique publique, initiée et mise en oeuvre par les États - génère d'importantes discussions depuis vingt ans. De tels projets de déplacement programmé furent adoptés comme solution aux dégradations environnementales et aux problèmes de pauvreté dans plusieurs régions et pays (Gemenne et al., 2012), incluant la Chine. Ces projets représentent un défi de taille pour les institutions publiques, et ceci de manière plus aiguë dans les pays émergents, qui souvent doivent produire, avec des moyens limités, des politiques d'une complexité et d'une ampleur non négligeable.

Suivant les meilleures pratiques de ce domaine, les décideurs sont censés tenir compte de l'éventail complet des facteurs susceptibles d'influer sur l'adaptation des communautés migrantes dans leur zone de destination, au risque de voir ces dernières retourner dans leur lieu d'origine. En effet, tout projet programmé de migration expose les migrants les plus vulnérables à divers risques, dont l'exclusion sociale et économique, l'appauvrissement matériel et la dislocation de la communauté. Ainsi, un défi majeur pour la recherche est de comprendre la façon dont les institutions peuvent favoriser/entraver cette adaptation et mitiger/aggraver les risques qui pèsent sur les migrants (Barnett et O'Neill, 2010).

\footnotetext{
1 Doctorant, département de recherches économiques, université du Ningxia, Yinchuan, Chine ; francois.dube.3@ulaval.ca

2 Doctorante, département de recherches économiques, université du Ningxia, Yinchuan, Chine ; yhj2061438@126.com

3 Directeur, centre de recherche sur le dévelopement de I'Ouest de la Chine, département de recherches économiques, université du Ningxia, Yinchuan, Chine ; hlj1963@163.com
} 
En Chine, les flux migratoires des provinces internes et des zones rurales vers les grands centres urbains ont monopolisé l'attention des chercheurs. Pourtant, les déplacements ayant pour cause des dégradations environnementales revêtent de plus en plus d'importance, notamment dans l'ouest du pays, où I'on observe de graves problèmes d'érosion des sols et de désertification. Situé au coeur de cet espace, dans une zone spécialement aride du plateau de Loess, la région autonome Hui du Ningxia constitue notre terrain d'enquête. Elle fut l'une des premières provinces à avoir initié, dès 1982, un processus officiel et programmé de migration environnementale.

Le présent article se focalise sur les facteurs qui peuvent expliquer les aboutissements et les conséquences de ces projets de déplacement de population. Pour ce faire, nous nous concentrerons sur les interactions entre les migrants et les institutions publiques chargées du processus de migration, et sur la façon dont celles-ci peuvent influer sur le bien-être des migrants pendant et après leur déplacement. Nous arguons que la gouvernance des institutions et le degré de participation des migrants peuvent jouer un rôle fondamental dans la mitigation des risques inhérents à de tels processus. Cela implique de considérer les migrants et les autorités dans une relation dynamique et interactive, de manière à expliquer les échecs ou les réussites des projets de migration environnementale. Nous verrons que les risques d'appauvrissement et d'exclusion ne sont pas des conséquences inévitables qui s'imposent aux migrants : Iorsqu'on leur en donne l'opportunité, ces derniers peuvent réagir, s'adapter, influencer et même améliorer les projets de déplacement, afin de faire valoir leurs intérêts et de mitiger les risques auxquels ils sont exposés.

Après avoir introduit les apports de la littérature sur les questions de la gouvernance inclusive et de la nature participative de la migration environnementale, nous présenterons les résultats d'une enquête empirique réalisée dans deux communautés migrantes de la région du Ningxia : les villages de Heshun et deYuanlong. Nous conclurons en examinant en quoi ces expériences nous renseignent sur l'importance de la gouvernance et de la participation pour l'adaptation et le bien-être des migrants environnementaux, et quelles leçons peuvent être tirées, à l'avenir, pour des projets similaires, en Chine et ailleurs.

\section{Migration environnementale, gouvernance et participation}

Dans le cadre de cette enquête, nous définissons les projets de migration environnementale ${ }^{4}$ comme toute politique initiée par les autorités centrales et mise en œuvre par les institutions régionales ou locales, visant à mitiger les problèmes écologiques et socio-économiques liés à la dégradation environnementale en déplaçant une partie ou la totalité d'une communauté d'une région jugée vulnérable vers une région apte à assurer la subsistance de cette communauté à long terme. Les autorités ont, par conséquent, le pouvoir de ralentir, accélérer ou forcer le déplacement et ainsi modeler les stratégies adoptées par

4 L'expression chinoise shengtai yimin est parfois traduite par " migration écologique " ou " relocalisation écologique ". Nous utilisons dans cet article l'expression " migration environnementale ". 
les individus et les communautés faisant face à des dégradations environnementales. En Chine, cette politique cherche à faire d'une pierre deux coups, à savoir rétablir les écosystèmes dégradés en réduisant la pression démographique et l'utilisation des terres, et combattre la pauvreté en favorisant le développement économique par la création de nouveaux milieux de vie pour les migrants, sur la base de moyens de subsistance axés sur le marché (West, 2009; Dickinson et Webber, 2007).

Ces projets peuvent être centralisés (les autorités jouant un rôle actif dans la sélection de la destination, la construction et l'aménagement du site, la distribution des terres, etc.), ou dispersés, les migrants étant libres de s'installer en différents endroits. La migration peut également se faire de manière volontaire ou forcée. Or, comme le soulignent Rogers et Wang (2006), dans un contexte chinois, ces distinctions ne sont ni évidentes, ni peut-être même pertinentes. Des migrants que l'on pourrait considérer, en théorie, volontaires font fréquemment l'objet de diverses pressions de la part des autorités locales, " rendant dans les faits la vie dans le lieu d'origine impossible ou irréaliste " (Rogers et Wang, 2006). Un examen des projets de migration en Chine doit s'efforcer de dépasser les examens formels des politiques et se concentrer sur l'expérience réelle des communautés migrantes.

Trop souvent, ces projets étatiques de déplacements de population aboutissent en un scénario perdant-perdant : les migrants sont fréquemment tout aussi, sinon plus vulnérables dans leur lieu de destination, et le problème environnemental, à l'origine de la migration, n'est pas pour autant réglé par leur départ. Plusieurs auteurs ont mis en évidence que les résultats prévus des projets de migration ne sont tout simplement pas au rendez-vous, en constatant que la raison d'être des projets est davantage liée à des objectifs de développement à court terme qu'à une véritable hausse de la qualité de vie des migrants (Weber et McDonald, 2004) et que les autorités se montrent en général très réticentes à intégrer les populations locales dans le processus (Nasritdinov et al., 2010). Cette situation semble particulièrement vraie en Chine, où ces déplacements plongent quelquefois les migrants dans une situation d'appauvrissement chronique, et où le taux d'échec de ces projets est extrêmement élevé (Liao et al., 2014).

Cependant, dans plusieurs cas, ces échecs ne sont ni d'origine externe (par exemple, la gravité de la dégradation environnementale) ni d'origine interne à la communauté migrante (le niveau de pauvreté prémigratoire), ils émanent de la mauvaise mise en oeuvre des projets de déplacement par les autorités en charge. Ces problèmes de gouvernance haussent la vulnérabilité de la communauté migrante et l'exposent ipso facto à de nombreux risques, déjà bien identifiés par le modèle de Cernea (1997). Ce dernier affirme que les principaux risques qui guettent les migrants lors de tels déplacements sont tout autant sociaux qu'économiques, et incluent entre autres la perte d'accès aux ressources naturelles (terres, eau), la baisse des revenus, la diminution de l'espace d'habitation, la marginalisation sociale, la désarticulation de la communauté, etc.

Face à ces risques d'exclusion et d'appauvrissement, il serait faux de considérer la communauté migrante comme passive. Nous nous inspirons ici de la littérature sur le concept des structures d'opportunités et de contraintes au sein desquelles évoluent les migrants (Martiniello et Rea, 2011). Ces structures 
sont influencées à la fois par le contexte institutionnel (principalement les environnements politico-légal et économico-institutionnel (Pécoud, 2004)) et par I'action des migrants (la mobilisation de leurs ressources sociales, économiques et culturelles). Si ces concepts sont le plus souvent utilisés pour analyser le parcours professionnel des migrants (voir Martiniello et al., 2009), ils sont tout aussi pertinents dans le contexte des projets de déplacement de population. En effet, ce double accent, sur le migrant et sur le cadre institutionnel, permet d'observer pleinement les interactions et les contraintes qui déterminent la gravité des risques inhérents à de tels projets.

L'Organisation internationale pour les migrations (OIM) insiste en effet sur I'importance de la gouvernance inclusive dans l'élaboration et la mise en oeuvre des déplacements programmés (2011). Pour cette dernière, la participation des migrants aux projets de migration peut, dans une certaine mesure, mitiger ces risques, et ce degré de participation est en grande partie déterminé par la nature inclusive de la gouvernance des projets. Confrontée à l'échec de plusieurs projets de déplacement, et aux violences résultant parfois de ces échecs, I'OIM n'hésite pas à recommander aux décideurs et aux institutions concernées d'accorder plus d'importance à la participation des migrants et des communautés ciblées.

De même, pour l'Organisation de coopération et de développement économiques (2012), le concept de participation est intimement lié à l'inclusion des populations cibles lors de l'élaboration des politiques publiques a priori, ou à tout le moins à la prise en compte de leur rétroaction a posteriori. Pour les projets de migration, cela suppose des échanges conséquents et significatifs entre les institutions et les populations devant être déplacées avant, pendant et après la migration. On peut juger de la nature participative d'un projet de migration sur la base des interactions entre les différentes parties prenantes, par lesquelles les institutions cherchent à modeler la réponse des migrants, alors que les migrants essayent d'influencer les institutions, afin de maximiser leurs gains et de garantir une transition réussie vers leur lieu de destination.

Selon l'approche retenue, les risques auxquels sont sujets les migrants sont le produit d'une interaction complexe entre la structure, qui comprend les politiques et les institutions qui restreignent ou créent des opportunités; les ressources en capital que les migrants peuvent mobiliser pour rétablir leurs moyens de subsistance; et leur contexte de vulnérabilité. Le présent article se focalise sur l'interaction entre ces trois éléments conceptuels, à savoir, dans quelle mesure la participation des communautés migrantes a pu (ou non) influencer la structure du projet de migration et améliorer leurs conditions de vulnérabilité ? Nous supposons que, dans tout processus de migration environnementale, une pleine et véritable participation des migrants à toutes les étapes du processus de migration joue un rôle essentiel dans la réduction des risques auxquels ils sont confrontés. Inversement, plus l'attitude des organismes gouvernementaux est stricte et exclusive à l'égard des demandes et des attentes des migrants, plus les chances que les migrants souffrent des vulnérabilités identifiées par Cernea sont grandes. 


\section{L'expérience chinoise en matière de migration envi- ronnementale}

L'essentiel des projets de migration environnementale du gouvernement chinois est localisé à l'ouest du pays, dans les provinces du Ningxia, Xinjiang, Qinghai, Gansu et de la Mongolie intérieure. Dans la plupart des cas, ces projets visent un double objectif (avec plus ou moins de succès, selon les cas) : arrêter ou ralentir la dégradation environnementale des régions vulnérables et aider les populations locales à se sortir du cercle vicieux de la pauvreté rurale. Concrètement, il s'agit de hausser le niveau de vie des migrants, à un niveau supérieur à celui proposé par leur habitat d'origine, en leur permettant de constituer une base de production autonome et intégrée au marché régional, le plus souvent en périphérie d'un centre urbain (Bartolome et al., 2000). Ce lien entre déplacement et développement se trouve au coeur des programmes de migration environnementale mis en œuvre en Chine, sous l'influence d'institutions comme la Banque mondiale, pour qui les déplacements écologiques constituent des opportunités de développement économique pour les régions qui accueillent les migrants.

D'après la typologie adoptée par Tan et Liu (2013), la politique chinoise en matière de migration environnementale peut s'appréhender au fil de trois phases principales. D'abord, de 1982 à 1993, le gouvernement central a lançé une première vague de projets visant à faciliter les déplacements de population afin de réduire la pauvreté dans certaines zones montagneuses dont la vulnérabilité environnementale était jugée élevée. Ces premières expériences ont été menées dans l'ouest de la Chine, en particulier, dans la préfecture Dingxi de la province du Gansu et dans le sud du Ningxia.

La deuxième phase, de 1994 à 2000, a consisté à élargir l'envergure des projets, en ciblant d'autres régions, et à bonifier les processus d'un véritable programme de lutte contre la pauvreté. Après une hausse considérable du niveau de vie en Chine suite aux réformes économiques, les catégories de population se trouvant toujours sous le niveau national de pauvreté absolue étaient alors concentrées dans les régions montagneuses durement affectées par les phénomènes de sécheresse, de désertification et autres dégradations environnementales. Les autorités centrales jugèrent alors qu'il était impossible de réduire le niveau de pauvreté de ces populations par des programmes de développement in situ, et le plan 80/7 fut adopté par le gouvernement central, dans le but de sortir 80 millions de paysans de la pauvreté en sept ans (en organisant leur déplacement si cela s'avérait nécessaire), et il ciblait 592 comtés dans dix-huit provinces chinoises ; dont 375 étaient situés dans l'ouest de la Chine (données du National Development and Reform Commission, 2007).

Dans la troisième phase, qui s'étend du début des années 2000 à aujourd'hui, les programmes de déplacement de populations se sont poursuivis essentiellement dans les mêmes régions non propices à la vie humaine et enclines à la désertification, et plus récemment, dans les régions devant être déclarées zones protégées et réserves naturelles, telles que les sources des grands fleuves au Qinghai et les zones jugées prédisposées au surpâturage en Mongolie intérieure. Cette période a également vu l'introduction du premier programme de compensation monétaire spécifiquement destiné aux migrants, alors qu'aupa- 
ravant les fonds disponibles étaient généralement liés aux programmes de lutte contre la pauvreté. Les projets disposant désormais de leur propre budget (trois à cinq milliards de yuans annuels) et intégrant de manière explicite des objectifs de développement économique et de protection environnementale, leur ampleur tend à s'étendre : certaines évaluations parlent du déplacement (volontaire ou forcé) d'environ dix millions de personnes dans les années à venir (Shi et al., 2007).

Ces projets d'envergure, parfois controversés, suscitèrent un intérêt non négligeable dans la littérature académique en Chine et à l'étranger. Au Ningxia spécifiquement, Xue, Liu et Tian (2013) se sont intéressés aux effets positifs des migrations sur les problèmes environnementaux, entre autres sur la déforestation, alors que Bai et Wen (2013) ont traité de la contribution positive des projets de déplacement à la réduction de l'écart de revenus entre le sud et le nord du Ningxia. La plupart des publications académiques ont privilégié les aspects économiques des projets de migration (Bao, 2006 ; Chen, 2007 ; Shi, 2008), mettant l'accent sur l'influence des déplacements sur le niveau de vie des migrants (Wilmsen et al., 2011 ;Tan et al., 2013). Dans cette région, comme dans d'autres provinces chinoises, ces enquêtes adoptent des méthodologies similaires, à savoir une comparaison entre la situation pré- et post-migratoire des ménages et des individus (Tang et al., 2012 ; Duan et McDonald, 2004 ; Dickinson et Webber, 2007).

Or, cette insistance sur une comparaison pré- et post-migratoire se désintéresse des aspects processuels des projets de migration. Les interactions entre les différents acteurs (essentiellement les autorités locales et les migrants) au cours des différentes phases de la migration (la conception du projet, la mise en œuvre et la gestion post-migratoire) ne sont ni intégrées dans les cadres conceptuels, ni spécifiquement examinées par les enquêtes empiriques. Si de telles approches sont aptes à évaluer la vulnérabilité des migrants, elles passent sous silence leurs réactivités face aux risques, ainsi que la manière dont l'interrelation entre les migrants et les autorités lors du processus de migration est en mesure d'influencer cette vulnérabilité. Plus qu'une question méthodologique, cette omission émane d'une hypothèse inhérente, quoique non explicite, aux cadres conceptuels de ces études, selon laquelle les migrants sont essentiellement des acteurs passifs des politiques de déplacement (Bartolome et al., 2000; Downing, 1996).

Plus récemment, certains auteurs ont tenté de remettre en cause cette hypothèse comme Tan et Liu (2013) sur la base de I'une des enquêtes empiriques les plus exhaustives réalisées dans la région du Ningxia. Ces auteurs mettent en évidence la façon dont les ménages réagissent aux dégradations environnementales en insistant sur le fait qu'il s'agit d'un choix stratégique très fortement conditionné par leur capital naturel, physique, social et financier, ainsi que par leur contexte institutionnel. De même, Gemenne (2009) et West (2009) invitent les chercheurs à intégrer la rétroaction des migrants à l'égard de leur environnement et des politiques mises en place par les autorités.

Les enquêtes tendent à démontrer que l'échec des déplacements serait en partie lié au faible degré de participation des migrants aux projets de migration. Dans leur examen des politiques de migration en Mongolie intérieure, Rogers 
et Wang (2006) notent que les migrants ne furent jamais en contact direct avec l'agence responsable du projet, ce qui engendra plusieurs malentendus et frictions au sujet des compensations et des modalités de déplacement. Foggin (2008) identifie le même phénomène dans le projet de déplacement de Sanjiangyuan, tout comme Banks, Richard, Ping et Zhaoli (2003) sur le plateau tibétain. Au Ningxia, les enquêtes chinoises et étrangères montrent que les résultats des programmes de restauration environnementale auraient été nettement plus positifs si les autorités avaient été davantage inclusives à l'égard de la rétroaction des migrants (Démurger et Wan, 2012; Liu, Li et al., 2008).

Dans cette logique, notre postulat central est que les migrants sont des participants actifs au processus migratoire, capables de formuler leurs demandes et de mobiliser leurs ressources (en tant qu'acteur rationnel) afin de maximiser leurs intérêts en influençant les institutions publiques. En Chine, bien que le processus de prise de décision du gouvernement central chinois soit encore guidé en grande partie par des principes de bureaucratisme et de centralisme, des espaces d'autonomie quant aux modalités locales d'application des politiques centrales tendent à émerger. Padovani (2004) et Grushke (2012) soulignent que grâce aux changements administratifs et politiques permettant un certain degré de décentralisation, les méthodes de mise en oeuvre des projets migratoires varient beaucoup en fonction de l'expertise, des capacités et de l'engagement des cadres et des responsables locaux. Ainsi, nous examinerons spécifiquement les deux facteurs clés qui forment l'interaction au coeur du processus migratoire : la nature inclusive des institutions publiques responsables de la conception et de la mise en oeuvre des projets de déplacement, et le degré de participation des communautés migrantes à ces mêmes projets. Cette approche est essentielle si nous voulons comprendre la " mécanique interne " des projets de déplacement, et non uniquement leurs résultats finaux.

\section{La région autonome Hui du Ningxia}

La région autonome Hui du Ningxia est une province montagneuse située au nord-ouest de la Chine qui se classe parmi les plus petites provinces chinoises, autant en population (6,25 millions) qu'en superficie (66 $000 \mathrm{~km} 2)$. Sur la base des indicateurs de revenus et d'espérance de vie, le Ningxia se situe sous la moyenne nationale, et est l'une des régions les moins industrialisées de Chine. Au coeur du plateau de Lœss (1050 mètres d'altitude), cette région est l'une des zones dont l'écologie et la capacité environnementale sont les plus vulnérables du pays. Les travaux de Liu et Wang (2001) montrent que la dotation en ressources naturelles de la province ne permettrait idéalement qu'une densité de 1,79 personnes $/ \mathrm{km}^{2}$, alors que la densité de population actuelle est de 89,9 personnes $/ \mathrm{km}^{2}$.

Dans le but d'alléger la pression démographique sur un environnement déjà fragilisé, de rétablir l'écosystème en cours de détérioration et d'éradiquer l'extrême pauvreté, le gouvernement du Ningxia fut l'un des premiers à mettre en place une politique de migration environnementale (Zhang et al., 2008). Celle-ci vise spécifiquement l'extrême sud de la région, appelé Xi-Hai-Gu, comprenant sept comtés catégorisés comme " pauvres " par l'État central : Xiji, Haiyuan, Guyuan, Pengyang, Jingyuan, Longde et Tongxin. Ces comtés 
comptent aussi la plus forte concentration de musulmans Hui : sur une population totale de 1,5 million, les deux tiers environ sont Hui (Song, 2000). Bien que généralement le niveau d'éducation des musulmans Hui soit moindre que celui des Han, leurs revenus ne diffèrent pas significativement, et la dégradation environnementale, les sécheresses ainsi que la pénurie d'eau affectent tout autant les deux groupes (Tan et Liu, 2013; Li et al., 2013). Se trouvant dans l'impossibilité d'irriguer les champs, les paysans dépendent des aléas des précipitations pour leur production alimentaire (I'expression locale " kaotianchifan " décrit cette situation, c'est-à-dire dépendre du ciel pour manger), ce qui explique pourquoi ces comtés furent déclarés, en 1972, comme l'une des régions les plus impropres à la subsistance de I'homme par le Programme des Nations unies pour le développement.

Depuis les trente dernières années, on estime que le gouvernement du Ningxia a organisé le déplacement de plus de 700000 paysans, majoritairement en provenance de ces comtés montagneux vers les comtés du nord (Yinchuan, Zhongwei et Wuzhong), où des systèmes d'irrigation utilisant l'eau du fleuve Jaune sont facilement accessibles. Nous avons sélectionné deux communautés représentatives de la plus récente phase de ces projets de migration environnementale, celle prévue par le Douzième plan quinquennal (2011-2015) du Ningxia5: le village de Heshun, qui a accueilli des villageois en provenance du district du Pengyang, en octobre 2011; et le village de Yuanlong, dont les habitants ont été déplacés du district de Longde, en mai 2012.

Ces deux projets de migration sont justifiés dans les documents officiels par une double logique, qui rappelle celle utilisée au niveau national, à savoir diminuer la pauvreté des populations du sud en les déplaçant vers des zones fertiles, et abaisser la pression démographique sur les zones arides et en pénurie d'eau. II ne s'agit pas d'une réponse à une catastrophe soudaine ou à une dégradation subite de l'environnement, mais plutôt d'un projet à long terme visant à alléger les problèmes écologiques en rééquilibrant la répartition de la population. Nous avons sélectionné ces villages en raison de leur comparabilité, autant en regard des modalités des projets de déplacement (dans les deux cas, organisé et mis en oeuvre par le gouvernement) que des circonstances prémigratoires (au point de vue des ressources économiques et sociales). En outre, ces deux villages présentent des résultats post-migratoires très différents : alors que Heshun est généralement considéré comme une expérience positive par les cadres locaux et les migrants, la communauté deYuanlong fait encore face à des problèmes relativement graves.

5 Le Douzième plan quinquennal du gouvernement du Ningxia prévoit l'investissement de 10,58 milliards de yuans pour mettre en œuvre le déplacement de 78800 familles (346 000 personnes) des régions centrales et méridionales du Ningxia vers le nord, en banlieue de la capitale régionale, Yinchuan. Ce plan touche 1655 villages dans les districts de Yuanzhou, Xiji, Longde, Jingyuan, Pengyang, Tongxin, Yanchi, Haiyuan et Shapotou. 


\section{Carte 1: Région autonome Hui du Ningxia}

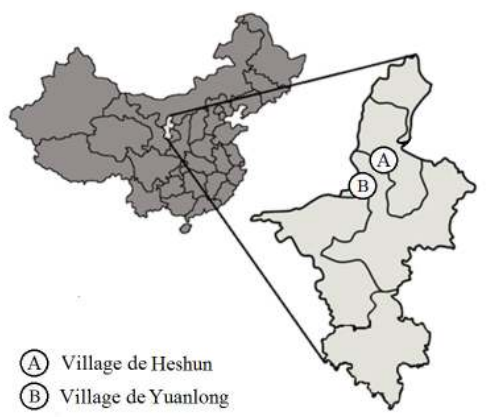

Cet article s'appuie sur des données recueillies lors de séjours de recherche sur le terrain, pendant I'hiver 2013, d'une durée de quatre jours, sur la base de méthodes participatives, en conformité avec les suggestions méthodologiques de I'OIM (2011). À un premier niveau, des questionnaires structurés furent distribués dans les communautés migrantes, au chef de famille, pour recueillir des données quantitatives sur la situation économique et sociale pré- et post-migratoire, mais surtout pour évaluer la participation des migrants, l'attitude des institutions locales et des cadres du village face à leur rétroaction, et l'influence perçue de cette participation sur leur situation présente. À cette collecte d'informations ont été ajoutées, des séances de discussions en groupe, réunissant plusieurs familles d'un(e) même quartier/rue, furent organisées. Dans les agences gouvernementales, des entretiens particuliers furent réalisés avec des responsables locaux, notamment les principaux cadres du village. En tout, 223 entrevues furent réalisées et les auteurs eurent la totale liberté dans le choix des sites et des répondants ${ }^{6}$.

\section{Participation à la conception prémigratoire du projet}

Les institutions impliquées dans les projets de migration à l'étude sont de deux ordres. Au niveau régional, les différents organes gouvernementaux du Ningxia étaient chargés d'établir et d'adopter les plans et les procédures régissant les projets, établissant les modalités de déplacements, incluant les barèmes de compensation, les mécanismes de consultations, etc. Tandis qu'au niveau local, les comités et les conseils des villages avaient en charge l'application et la mise en oeuvre de ces procédures. Nous verrons que, dans le cas qui nous intéresse, c'est précisément l'écart entre les procédures, les cadres adoptés au niveau régional et leur mise en opération sur le terrain qui retiendra notre attention.

Un examen des plans initiaux adoptés au niveau régional, ainsi que nos discussions avec les cadres locaux des deux villages, montrent que ces derniers avaient été chargés de transmettre à chaque famille les détails et les modalités 
du projet de migration environnementale avant le déplacement, et de recueillir les demandes, les suggestions et les opinions des migrants à ce sujet. Or, nos résultats révèlent que cette procédure fut en majeure partie formelle et superficielle, et que les demandes et les suggestions des migrants ne furent pas examinées de manière adéquate ni par les cadres locaux ni par l'organe régional responsable de la migration.

Les données recueillies par questionnaire confirment ce point : à la question "Avant le déplacement, le gouvernement a-t-il sollicité vos suggestions et demandes concernant le projet de migration ? Si c'est le cas, vos suggestions et les demandes ont-elles été prises en compte par le gouvernement ? ", les résultats de l'enquête mettent en évidence des tendances semblables dans les deux villages retenus, seulement $28,4 \%$ des migrants ont répondu " oui ", et parmi eux, uniquement $10,5 \%$ considèrent à différents degrés que leurs suggestions ont été adoptées et mises en oeuvre par les autorités chargées de concevoir le projet de migration.

Le travail de diffusion des informations fut loin d'être idéal, certains migrants se disaient encore peu informés des détails spécifiques de la politique de migration lors des visites sur place. Dans le village de Heshun, 50,2 \% des migrants affirment comprendre " très bien " et " assez bien " le projet de migration, alors que dans le village de Yuanlong, ils sont seulement 34,9\% à avoir choisi l'une de ces deux options. Parmi les demandes des migrants avant le déplacement, certains villageois avaient exigé de pouvoir visiter le nouveau village afin d'évaluer les conditions de vie dans le lieu de destination. Pourtant, dans les deux cas étudiés, les autorités locales ont refusé d'organiser de telles visites pré-migratoires.

Les témoignages indiquent que cette situation ne correspond aucunement à la forte volonté de participation des migrants. Plusieurs ont affirmé avoir tenté de faire valoir leurs doléances (de manière autonome et sans invitation des cadres locaux), pour se voir finalement refuser toute consultation. II apparaît donc que le faible taux de participation relève non pas d'une supposée apathie des communautés migrantes, mais bien plus du non-respect, par les autorités locales, des modalités de consultation des migrants prévues dans les projets de déplacement.

\section{Participation à la mise en oeuvre du projet}

Concernant la phase de mise en oeuvre, notre enquête s'est concentrée sur la participation des migrants en fonction de trois aspects de leur vie dans leur nouvel habitat, à savoir les sources de revenus, I'accès aux ressources en eau et le logement. Dans le dernier cas, le projet de déplacement des deux communautés tel qu'adopté au niveau régional prévoyait la réinstallation des familles migrantes dans des logements standards de $54 \mathrm{~m}^{2}$, dont la qualité était grandement supérieure aux logements délabrés de leur lieu d'origine.

Cependant, nos discussions avec les migrants révèlent un certain nombre de problèmes. L'allocation des logements, en particulier, s'est faite non pas en fonction du nombre d'individus, mais sur la base du livret de famille, ce qui engendra des situations aberrantes dans les deux communautés. En effet, 
dans le cas où les parents et les enfants sont tous inscrits sur le même livret de famille, la famille n'a droit qu'à un seul logement, indépendamment du nombre de personnes. Pour les familles nombreuses, où certains enfants vivaient séparément des parents dans le village d'origine, cela peut représenter une sérieuse dégradation de la qualité de vie. C'est le cas, par exemple, de la famille Wu, du village Yuanlong, qui se compose de sept personnes : alors que ces derniers habitaient trois maisons avant la migration, ils ont été obligés de cohabiter dans un seul logement standard de $54 \mathrm{~m}^{2}$ dans leur nouveau village.

Pour résoudre ce problème, certaines familles ont cherché à construire à leurs propres frais une seconde habitation sur la terre qui leur a été attribuée dans le nouveau village, mais les cadres locaux de Yuanlong ont catégoriquement refusé, menaçant de détruire toute maison ainsi construite. Lorsqu'elles ont été interrogées, les autorités locales étaient peu enclines à accepter de telles initiatives des migrants, prétextant que la situation deviendrait "ingérable " et que l'espace était restreint. De plus, elles ont ajouté qu'une telle situation risquerait d'engendrer des conflits sociaux du fait des disparités évidentes de moyens entre les familles. Notre enquête montre que, malgré le fait que plusieurs familles font face à ce problème, les cadres deYuanlong demeurent intraitables.

Dans le village de Heshun, par contre, si les problèmes sont les mêmes, l'attitude des autorités est radicalement différente. Les villageois affirment que les cadres du village se préoccupent de ces problèmes. Certaines familles ont donc reçu l'autorisation d'agrandir leur logement, ou même de construire à leur frais une seconde maison sur leurs nouvelles terres. De plus, dans le cas des familles nombreuses comprenant des personnes âgées, les autorités ont favorisé le déplacement de ces dernières dans des maisons de retraite situées non loin du village, ou dans des logements voisins où certaines pièces sont vides, suivant une formule de cohabitation.

Les sources de revenus et les moyens de subsistance sont aussi une question fondamentale ayant une incidence sur l'adaptation et l'intégration des migrants dans leur nouveau milieu. Dans les cas étudiés, les moyens de subsistance prémigratoires des villageois reposaient principalement sur une agriculture pluviale extensive ; la superficie de leurs terres était alors grande. Conformément à la politique de migration, une parcelle de terres irriguées fut allouée aux migrants. Et alors que les familles migrantes, avant le déplacement, disposaient de terres d'une superficie moyenne par habitant d'environ cinq $\mathrm{mu}\left(3330 \mathrm{~m}^{2}\right)$, consistant majoritairement en des terres arides, après la migration, la superficie moyenne par famille est passée à environ 1,68 mu (1 $\left.118 \mathrm{~m}^{2}\right)$, dont 1,56 mu (1038 $\left.\mathrm{m}^{2}\right)$ de terres irriguées. Les migrants reçurent dans le même temps les droits d'utilisation des terres, incluant les droits de gestion contractuelle, de sous-traitance, de location, d'échange, de transfert et d'autres formes de coopération. Or, notre enquête a révélé que la gouvernance des autorités par rapport aux migrants et ses effets sur leurs moyens de subsistance diffèrent dans les deux villages.

Dans le village de Heshun, les autorités ont alloué à chaque famille migrante : une serre agricole, en plus d'une parcelle de $2 \mathrm{mu}\left(1332 \mathrm{~m}^{2}\right)$. De plus, des séances de formation furent organisées pour enseigner aux migrants les techniques agricoles modernes et leur fournir une variété de compétences dans ce domaine. Lors de notre visite sur place, la majorité des répondants étaient au 
travail dans les serres, qui sont utilisées à pleine capacité ; elles produisent deux récoltes de légumes par année, principalement des tomates et des tubercules. Les membres du personnel professionnel et technique effectuent régulièrement des visites sur les lieux afin d'inspecter et de corriger au besoin les mauvaises pratiques, même plusieurs mois après la fin de la formation initiale ; $80 \%$ des migrants se disent à ce propos pleinement confiants quant au futur de leurs activités agricoles.

Au sein du village Yuanlong, les autorités concernées ont alloué aux familles une parcelle de terres irriguées de $1 \mathrm{mu}\left(666,7 \mathrm{~m}^{2}\right)$, incluant aussi des séances de formation. Or, peu de temps après la migration, la majorité des migrants décidèrent de céder les droits d'exploitation de leur terre à une compagnie tierce, pour une somme très modique (quelque 200 yuans par année). Lorsqu'ils ont été questionnés sur les raisons d'un tel transfert, la plupart des migrants ont révélé qu'ils ne comprenaient que très peu ce genre d'agriculture, spécialement les systèmes d'irrigation, et craignaient de commettre des erreurs. Ainsi, plusieurs se retrouvent sans source de revenus suffisante. Il leur est difficile de trouver un travail d'appoint en ville, en raison de leur manque de compétences techniques. Dans une situation où les dépenses des familles ont relativement augmenté par rapport à leur situation pré-migratoire, l'incapacité des migrants à exploiter eux-mêmes leurs terres est un handicap sérieux à leur intégration dans leur nouveau milieu, et une majorité d'entre eux se montrent pessimistes par rapport au futur de leur communauté.

Sur la base de nos discussions avec les migrants, nous avons pu constater qu'ils étaient pour la plupart désireux de suivre une formation liée aux techniques agricoles, et que dans les deux cas, les autorités ont organisé des activités de formation. Toutefois, le niveau de participation des migrants diffère, il s'élève à $71 \%$ à Heshun et n'est que de $26 \%$ à Yuanlong. Les raisons de cette faible participation sont à trouver dans le fait que la plupart des migrants de Yuanlong considèrent que la formation ne répond pas à leur besoin (notamment leur difficulté à passer d'une agriculture pluviale à des systèmes d'irrigation), et que la formation ne comprend pas de volet pratique, se limitant à des connaissances théoriques. Lors de nos entretiens, les cadres de Yuanlong ont reconnu que la plupart des migrants étaient réticents à se joindre à la formation, et qu'ils ont tenté d'augmenter leur participation en offrant des petits cadeaux en guise de mesure incitative. Sans surprise, de tels ajustements ne donnent que très peu de résultats, car ils ne s'attaquent aucunement aux sources du problème : la formation visiblement ne répond pas aux attentes et aux besoins de la population.

Enfin, le lieu d'origine des migrants étant situé en région montagneuse aride, où les précipitations annuelles sont particulièrement rares, un approvisionnement en eau fiable et facile d'accès est considéré comme une amélioration de la qualité de vie dans leur nouvelle communauté. Dans leur village d'origine, les besoins en eau pour la production agricole dépendaient de la pluie, alors que les besoins personnels étaient satisfaits par de l'eau potable achetée à l'extérieur du village, ce qui représentait une dépense importante pour les ménages ruraux. II n'est pas étonnant que le gouvernement ait insisté sur la construction d'installations d'eau potable dans les lieux de destination. Dans les deux villages, la majorité de la population peut désormais utiliser l'eau du robinet, et les besoins 
pour la production agricole sont principalement satisfaits par les abondantes ressources en eau du fleuve Jaune. Dans les deux cas, plus de la moitié des immigrants considèrent généralement que la situation actuelle répond à leurs besoins.

Toutefois, l'enquête montre aussi que dans le village de Yuanlong, bien que le système d'eau potable soit en service, les interruptions sont très courantes, environ trois à cinq fois par mois et durent plusieurs jours. Elle souligne aussi que l'équipement de filtration d'eau est relativement simple et ne répond pas aux normes sanitaires. En conséquence, plusieurs migrants ont exprimé leur inquiétude quant à la qualité de l'eau du robinet, mais ces griefs ont été ignorés par les cadres locaux. De plus, les villageois de Yuanlong affirment que les autorités avaient promis de couvrir les frais d'eau des ménages pendant la première année suivant leur migration, afin de faciliter une transition réussie, mais que cette promesse est également restée lettre morte.

En outre, les migrants se plaignent d'un grave problème de communication, les autorités locales de Yuanlong n'ayant pas correctement informé les familles des frais liés à l'utilisation de l'eau courante. En conséquence, plusieurs ménages ont fait une utilisation prolongée de l'eau courante pour arroser leur jardin, causant une augmentation significative de leurs dépenses. La situation est relativement meilleure dans le village de Heshun, à la fois en termes de qualité de l'eau et du nombre d'interruptions. De plus, les migrants de Heshun disent avoir été informés par les autorités locales du coût de l'eau courante et de la manière d'en faire une utilisation modérée.

\section{Participation à la gestion postmigratoire du projet}

Les résultats de notre enquête indiquent que, suite à la migration, le degré d'implication des migrants dans la gestion des affaires publiques subit une importante hausse. Avant la migration, la plupart des villageois vivaient de manière éparse et participaient rarement aux affaires publiques et à l'administration de leur communauté, alors qu'après le déplacement, leur degré de participation a augmenté de manière considérable. Or, en raison des différences d'attitude et d'efficacité des cadres des deux communautés, cette participation engendra différents résultats.

Dans le village de Heshun, suite à la migration, près de $46,3 \%$ des personnes affirment participer régulièrement ou occasionnellement à l'assemblée populaire de leur village, alors qu'avant la migration, ce taux n'était que de 23,2 \%. De plus, les migrants disent recourir plus souvent aux cadres du village qu'auparavant lorsqu'ils rencontrent une difficulté : la proportion des migrants ayant eu recours à leur aide a fait un bond significatif, passant de $4,8 \%$ à $32,2 \%$. Dans le village de Yuanlong, l'amélioration de la participation est beaucoup moins impressionnante. Environ $27,8 \%$ des migrants disent assister régulièrement ou occasionnellement à l'assemblée populaire locale, alors qu'avant la migration, cette proportion était de $20,6 \%$. Quant à leur recours à l'aide des cadres du village, nos données montrent une augmentation de seulement 5,3\%. 
Pour expliquer leur participation moindre à la gestion du village, les migrants de Yuanlong ont affirmé avoir vécu de mauvaises expériences. La plupart du temps, lorsqu'ils se rendent à la mairie pour rapporter leurs problèmes, l'attitude du personnel est en général indifférente, voire hostile, et les solutions proposées ne sont aucunement à la hauteur des problèmes rencontrés. Sur la base des discussions, il semble que résoudre le moindre petit souci demande de la part des migrants de grands efforts administratifs, ainsi des visites répétées au bureau. De plus, plusieurs migrants soulignent le manque d'intégrité des cadres du village de Yuanlong. Certains migrants ont indiqué que même si leurs dossiers étaient éligibles à une aide gouvernementale, il était difficile de toucher les montants sans faire jouer leurs relations au sein de la mairie locale. Ce genre de problèmes s'ajoute à d'autres cas que les migrants de Yuanlong considèrent comme des abus de pouvoir de la part des autorités du village.

Ces différences entre les deux villages dans la gestion post-migratoire des affaires communautaires se reflètent concrètement dans le degré général de satisfaction des migrants à l'égard de leur expérience de migration. Lorsqu'ils furent questionnés sur la qualité du travail des cadres locaux, les migrants de Heshun se déclarèrent à $89,8 \%$ assez ou très satisfaits, alors que dans le village de Yuanlong, cette proportion n'est que de 52,9 \%. À Heshun, 70,5 \% des migrants se sont déclarés satisfaits de leur situation post-migratoire, et pour $13,9 \%$ non satisfaits ; à Yuanlong, même si la satisfaction est relativement élevée $(53,6 \%)$, le fort taux de migrants non satisfaits $(35,5 \%)$ témoignent des problèmes décrits précédemment.

\section{La carence participative}

Dans les deux villages étudiés, la politique de migration environnementale du Ningxia fut mise en œuvre par les autorités locales d'une façon unilatérale. Avant la migration, le degré de participation des migrants à la conception et à la planification du projet de migration environnementale fut faible, sinon nul. Même si, dans les deux cas, le plan de migration adopté par les organes régionaux prévoyait sur papier une phase de consultation, ainsi que la prise en compte des demandes des villageois. Les différentes facettes du projet furent essentiellement organisées et pilotées par les cadres locaux et sans participation des migrants eux-mêmes. II apparaît clairement que - même si les plans officiels prévoient des mesures d'inclusion et de participation - les cadres locaux sont réfractaires à les mettre en oeuvre et tendent à considérer les migrants uniquement comme des bénéficiaires passifs des politiques de déplacement environnemental, et non comme des acteurs participants.

Nous relevons la présence d'un décalage constant, à toutes les étapes du processus de migration, entre d'une part les lois, politiques et procédures telles qu'elles furent conçues sur papier et adoptées par le gouvernement régional, et d'autre part leur mise en œuvre dans les communautés migrantes. Ce décalage, nous l'avons souligné, a eu des conséquences directes et néfastes sur les communautés migrantes elles-mêmes, notamment en matière de logement, d'emploi et d'accès aux ressources en eau. Cela est d'autant plus manifeste que les plans de déplacement prévoyaient sur le papier des mesures visant à atténuer chacune des lacunes observées sur le terrain. Les projets eux-mêmes 
sont bien plus à blâmer que les personnes responsables de la mise en oeuvre imparfaite des procédures.

Cette mauvaise installation des projets se reflète de manière évidente dans les phases de mise en place et de gestion postmigratoire, où le faible degré de participation des migrants du village de Yuanlong eut des effets négatifs considérables. L'expérience de Yuanlong montre qu'un processus de migration qui ne comporte pas de participation directe des migrants est à même d'entraîner des situations aberrantes qui peuvent être difficilement justifiables. Allouer des logements systématiquement de même taille à des familles dont les membres varient de deux à sept n'est pas raisonnable, tout comme offrir une formation agricole qui se limite à quelques cours théoriques sans répondre aux besoins concrets des migrants. Les entrevues avec les institutions responsables de la mise en oeuvre du projet soulignent que ce manque de participation est justifié afin "d'éviter les ennuis inutiles " ou de " devoir respecter les délais fixés par le gouvernement régional ». Loin de réaliser les objectifs initiaux de la politique, visant à améliorer les conditions de vie des migrants, l'expérience du village de Yuanlong indique que cette gouvernance unilatérale tend à avoir un effet contraire, à savoir aggraver les risques auxquels sont sujets les migrants.

Notre enquête dans le village de Heshun révèle que les autorités locales, notamment les cadres villageois, sont capables de mitiger ces risques en adoptant une attitude participative et inclusive à l'égard des demandes des migrants. Ce mode de gouvernance inclusif, permettant un certain espace de flexibilité dans l'application des politiques, est apte à permettre des progrès beaucoup plus substantiels dans la réalisation des objectifs originaux du projet. Les demandes des migrants du village de Heshun furent dans une large mesure intégrées par les institutions, atténuant ainsi les risques liés aux pertes matérielles en termes de logement, de sources de revenus et d'approvisionnement en eau.

II est toutefois difficile d'expliquer cette différence entre les deux cas retenus : pourquoi le décalage est-il si apparent dans le cas de Yuanlong, alors que les cadres de Heshun se sont montrés plus fidèles à la lettre et à l'esprit des projets migratoires adoptés au niveau régional. Est-ce le manque de professionnalisme, de compétences ou tout simplement le désintérêt des autorités locales? Les témoignages recueillis auprès des cadres des villages n'offrent que peu $\mathrm{d}$ 'indices à cet égard. Une enquête traitant spécifiquement de cet aspect serait plus apte à répondre à ces questions, notamment en centrant l'analyse sur les qualifications, la formation et l'expérience des cadres locaux.

Nos résultats confirment certains résultats d'enquêtes menées sur les mécanismes internes d'élaboration et de mise en oeuvre des politiques publiques en Chine, notamment dans le cadre de migrations environnementales. Récemment, certains auteurs commeTang et al. (2012), Grushke (2012) et Foggin (2011), entre autres, ont identifié les effets positifs d'une véritable participation communautaire et de l'appropriation du projet par les populations locales. Ces derniers arrivent à la conclusion que l'élément clé de la réussite des projets de migration environnementale est " de travailler au rythme de la communauté, et non pas au rythme des parties externes, et ce qui est plus important encore, de rechercher et de donner la possibilité aux voix locales de se faire entendre aussi souvent que 
possible " (Foggin, 2008). L'OIM (2011) abonde dans le même sens, soulignant que les projets de déplacement les plus réussis furent ceux où le processus de prise de décision fut le plus participatif.

Ainsi, il apparaît comme nécessaire de dépasser le cadre d'analyse traditionnel des politiques de migration environnementale, qui cherche à évaluer les politiques sur la base d'un inventaire postmigratoire des pertes et des gains matériels. Il est plus utile d'examiner en quoi la vulnérabilité des migrants et le succès d'un projet de migration dépendent de la manière dont la gouvernance du projet influence ces résultats postmigratoires. II ressort de notre enquête qu'une approche qui ne s'appuie pas sur une participation des individus et des familles migrantes n'est pas à même de réaliser l'effet bénéfique que peuvent produire ces interactions. Seule une gouvernance inclusive à l'égard des migrants peut résoudre ce problème. Or, pour ce faire, ces derniers doivent participer au processus de prise de décision des autorités, par l'entremise de séances d'information, de réunions, de discussions publiques, etc. Cela suppose que les autorités locales s'éloignent des modèles traditionnels de gestion top-down et apprennent à réaliser des compromis, voire à partager le pouvoir, ce qui, en Chine, semble relativement difficile.

\section{Conclusion}

Les recherches dans le domaine de la migration environnementale doivent dépasser les approches d'évaluation pour pleinement comprendre la complexité qui caractérise le rôle des institutions dans les projets de déplacement. Les résultats présentés dans cette enquête, sur la base d'une étude de terrain s'intéressant au processus de migration, et non uniquement aux aboutissements du projet, indiquent que la nature inclusive de la gouvernance des projets de déplacement est en grande partie liée à une meilleure mitigation des risques auxquels sont exposés les migrants. Cela confirme notre postulat selon lequel les modèles réduisant les migrants à un rôle passif doivent être remis en question et sont somme toute peu utiles pour expliquer les succès ou les échecs de projets de déplacement de population.

Une telle approche permet aussi de mettre en valeur la vision des migrants, qui se révèle souvent critique quant à la légitimité mêmede tels projets de déplacement de population. Dans un contexte où les responsables politiques et certains universitaires chinois tolèrent mal les critiques à l'égard de ces projets (Gemenne, Brücker et lonesco, 2012 ; Wang, Song et Hu, 2011), il est essentiel de garder à l'esprit que d'autres solutions sont disponibles pour restaurer l'environnement tout en développant l'économie locale des régions concernées. Ces solutions peuvent inclure, entre autres le développement de l'écotourisme et le partage des bénéfices des projets de développement. Les études ancrées sur le terrain, qui replacent au centre de leurs analyses les migrants, leur autonomie et leurs stratégies pourraient s'avérer extrêmement intéressantes. 


\section{Références bibliographiques}

Bai Xuejun and Wen Li (2013) An Effectiveness Analysis on Policy of Eco-migration in Ningxia and Reflections of Constructing a New Harmonious and Prosperous Ningxia, Research of Agricultural Modernization, 2, pp. 159-162.

Banks Tony et al. (2003) Community-Based Grassland Management in Western China Rationale, Pilot Project Experience, and Policy Implications, Mountain Research and Development, 23 (2), pp. 132-140.

Bao Zhiming (2006) The Definition, Category and Some Other Issues about Ecological Migration, Journal of The Central University For Nationalities, 1, pp. 27-39.

Barnett Jon and O'Neill Saffron (2010) Maladaptation, Global Environmental Change, 20, pp. 211-213.

Bartolome Leopoldo Jose et al. (2000) Displacement, Resettlement, Rehabilitation, Reparation, and Development, World Commission on Dams. Secretariat of the World Commission on Dams, Cape Town, 86 p.

Cernea Michael (1997) The Risks and Reconstruction Model for Resettling Displaced Populations, World Development, 25 (10), pp. 1569-88.

Chen Guojie (2007) Strategic Thoughts and Suggestions of Eco-migration in China, Decision and Consulting Letter, 5, pp.1-4.

Démurger Sylvie and Wan Haiyuan (2012) Payments for Ecological Restoration and Internal Migration in China: The Sloping Land Conversion Program in Ningxia, IZA Journal of Migration, 1 (10), $32 \mathrm{p}$.

Dickinson Debbie and Webber Michael (2007) Environmental Resettlement and Development on the Steppes of Inner Mongolia, PRC, Journal of Development Studies, 43 (3), pp. 537-561.

Downing Theodore (1996) Mitigating Social Impoverishment when People are Involuntarily Displaced, in Christopher McDowell (Éd.), Resisting Impoverishment:Tackling the Consequences of Development-Induced Impoverishment, Oxford, Bergham Books, pp. 132-140.

Duan Yuefang and McDonald Brooke (2004) Involuntary Resettlement as an Opportunity for Development: The Application of 'Resettlement with Development' in the People's Republic of China, Working Paper,14, Melbourne, Melbourne University Private, pp. 82-102.

Foggin Jean-Marc (2008) Depopulating the Tibetan Grasslands, Mountain Research and Development, 28 (1), pp. 26-31.

Foggin Jean-Marc and Torrance-Foggin Marion (2011) How Can Social and Environmental Services Be Provided for Mobile Tibetan Herders? Collaborative Examples from Qinghai Province, China, Pastoralism: Research, Policy and Practice, 1(21), pp. 1-21.

Gemenne François, Brücker Pauline and Ionesco Dina (2012) The State of Environmental Migration 2011, Paris, Institut du développement durable et des relations internationales, $132 \mathrm{p}$.

Gemenne François (2009) L'environnement, nouveau facteur de migrations ?, in Jaffrelot Christophe et Lequesne Christian (Éds.), L'enjeu mondial : les migrations, Paris, Presses de Sciences Po, 311 p. 
Grushke Andreas (2012) Tibetan Pastoralists in Transition. Political Change and State Interventions in Nomad Societies, in Hermann Kreutzmann (Éd.), Pastoral Practices in High Asia. Agency of 'Development' Effected by Modernisation, Resettlement and Transformation, Springer, Dordrecht, pp. 273-289.

Hong Tang et al. (2012) Ecological Migration Effects on the Tianchi Scenic Area in Xinjiang: From the Perspective of Migrant Farmer Families, Journal of Arid Land, 4 (1), pp. 95-104.

Iredale Robyn, Bilik Naran and Guo Fei (2003) China's Minorities on the Move. Selected Case Studies, Armonk, New York, London, M.E. Sharpe, 183 p.

Jianguo Liu et al. (2008) Ecological and Socioeconomic Effects of China's Policies for Ecosystem Services, Proceedings of the National Academy of Sciences of the United States of America, 9105, pp. 9477-9482.

Li Lihua et al.(2013) Migration, Remittances, and Agricultural Productivity in Small Farming Systems in Northwest China, China Agricultural Economic Review, 5 (1), pp. 5-23.

Liao Chuan et al. (2014) Following the Green: Coupled Pastoral Migration and Vegetation Dynamics in the Altay and Tianshan Mountains of Xinjiang, China, Applied Geography (46), pp. 61-70.

Liu Yanhui and Wang Qiang (2001) Suitable Distribution of China's Population, China Population, Resources and Environment [in Chinese], 11(1), pp. 34-37 .

Martiniello Marco et Rea Andrea (2011) Des flux migratoires aux carrières migratoires, SociologieS, Dossiers, Migrations, pluralisation, ethnicisation des sociétés contemporaines, [en ligne] consulté le 06/03/2015. URL : http://sociologies.revues.org/3694.

Martiniello Marco et al. (2009) Nouvelles migrations et nouveaux migrants en Belgique, Société et Avenir, Gent, Academia Press, 339 p.

Nasritdinov Emil et al. (2010) Environmental Migration: Case of Kyrgyzstan, Environment, Forced Migration and Social Vulnerability, pp. 235-246.

National Development and Reform Commission (2007) China's National Climate Change Programme, [en ligne] consulté le 11/06/2015. URL : http://en.ndrc.gov. cn/newsrelease/200706/P020070604561191006823.pdf

Organisation de coopération et de développement économiques, Thoreau Cécile and Liebig Thomas (2012) Settling in: OECD Indicators of Immigrant Integration, [en ligne] consulté le 11/06/2015. URL : www.oecd.org/els/mig/Secretariat_ Press_3Dec2012_Final\%20REV.pdf

Organisation internationale pour les migrations (2011) IOM Project Handbook, Suisse, Genève, $440 \mathrm{p}$.

Padovani Florence (2004) Migrations forcées et grands travaux hydrauliques en Chine : le cas du barrage des Trois Gorges, Géocarrefour, (79), pp. 27-34.

Pécoud Antoine (2004) Réseaux, ethnicité et institutions dans les économies immigrées, Hommes et Migrations, 1250, pp. 13-23.

Rogers Sarah and Wang Mark (2006) Environmental Resettlement and Social Dis/Rearticulation in Inner Mongolia, China, Population and Environment, 28 (1), pp. 41-68. 
Shi Guoqing and Zhou Jian (2007) Protection of Rights and Interests and Government's Responsibilities: a Case Study of the Tarim River Ecological Migration as an Example, Jilin University Journal Social Sciences, (47), pp. 78-86.

Shi Su (2008) Living Condition and Social Adaptation of Sanjiangyuan Eco-migration: Taking Case of Eco-migration in Golmud city from Changjiang Source, Tibetan Studies, 4, pp. 93-102.

Song Peng (2000) Strategies for the 'Aid-the-poor' Migration Program in Ningxia Autonomous Region, Human Geography, 15 (5), pp. 47-51.

Tan Yan and Liu Xuchun (2013) In-situ Adaption and Out-Migration: Enablers and Constraints Among Rural Households of the Mountainous Region of Southern Ningxia, Northwest China, XXVII IUSSP International Population Conference, Busan, $34 \mathrm{p}$.

Tan Yan, Zuo Alec and Hugo Graeme (2013) Environment-related Resettlement in China: A Case Study of the Ganzi Tibetan Autonomous Prefecture of Sichuan Province, Asian and Pacific Migration Journal, 22 (1), pp. 77-107.

Wang Zongming, Song Kaishan and Hu Liangjun (2011) Response to "Rethinking Ecological Migration and the Value of Cultural Continuity", AMBIO A Journal of the Human Environment, 40 (1), pp. 100-101.

Webber Michael and McDonald Brooke (2004) Involuntary Resettlement, Production and Income: Evidence from Xiaolangdi, PRC, World Development, 2004 (4), pp. 673-690.

West Jennifer (2009) Perceptions of Ecological Migration in Inner Mongolia, China: Summary of Fieldwork and Relevance for Climate Aadaptation, Oslo, CICERO, $22 \mathrm{p}$.

Wilmsen Brooke, Webber Michael and Duan Yuefang (2011) Involuntary Rural Resettlement: Resources, Strategies, and Outcomes at the Three Gorges Dam, China, The Journal of Environment and Development, 20, pp. 355-380.

Xue Xinle, Liu Zhiping and Tian Guojiang (2013) Study on Ecological Restoration and Management Problems of Ecological Out-migration Area in Longde County of Ningxia, Journal of Gansu Forestry Science andTechnology, 2011 (4), pp. 53-54.

Zhan Xiaolin and Bingyuan Tao (2008) Study on the Value Estimation and Compensation Mechanism of Ecological Migration in Sanjiangyuan Area, Ascent, (6), pp. 66-69.

Zheng Yi, Jiahua Pan and Xiaoyu Zhang (2013) Relocation As a Policy Response to Climate Change Vulnerability in Northern China, World Social Science Report 2013, OECD, pp. 234-242. 


\section{François N. Dubé, Yang HaiJuan et Huang LiJun}

\section{..: Participation des populations aux projets programmés de migration environnementale dans le Ningxia (Chine)}

Les projets de migration programmée de populations pour cause de dégradations environnementales représentent un véritable défi pour les institutions publiques, et impliquent plusieurs risques pour les migrants eux-mêmes, particulièrement dans les pays émergents comme la Chine. Cet article vise à explorer l'influence de la gouvernance des institutions et de la participation des migrants sur la mitigation de ces risques. Sur la base d'une enquête empirique réalisée dans deux villages de la province du Ningxia, nous considérons la relation dynamique et interactive entre les migrants et les autorités locales, et constatons que la nature participative (ou non) des institutions peut jouer un rôle fondamental dans la réussite ou l'échec des projets de déplacement. Ainsi, les risques d'appauvrissement et de désarticulation sociale ne sont pas des conséquences inévitables qui s'imposent aux migrants : lorsqu'on leur en donne l'opportunité, ces derniers peuvent réagir, s'adapter, influencer et même améliorer les projets, afin de faire valoir leurs intérêts et de mitiger les risques auxquels ils sont exposés.

\section{Governance and migrant participation in environmental migration projects in Ningxia (China)}

Policies of forced migration of populations due to environmental degradation pose a real challenge for public institutions, and expose migrants themselves to a certain number of potential risks, particularly in emerging countries such as China. This article aims to explore the contribution of institutional governance and migrant participation to the mitigation of these risks. Based on an empirical survey conducted in two villages in Ningxia autonomous province of China, we consider the dynamic and interactive relations between migrants and local government, and find that the (non-)participatory nature of local institutions plays a fundamental role in determining the success or failure of the migration policies. Thus, the risks of impoverishment and social dislocation are not inevitable consequences imposed on migrants: when given the opportunity, they can respond, adapt, influence and even improve projects in order to promote their interests and mitigate the risks to which they are exposed.

\section{... Gobernabilidad y participación de los migrantes en los proyectos de migración ambientales en Ningxia (China)}

Las políticas de gestión de la migración forzoso de poblaciones debido a la degradación ambiental representan un verdadero desafío para las instituciones públicas, y exponen a los migrantes a numerosos riesgos potenciales, especialmente en los países emergentes como China. Este artículo tiene como objetivo explorar la contribución de la gobernabilidad institucional y de la participación de migrantes a la atenuación de estos riesgos. Basado en un estudio empírico realizado en dos aldeas de la Región Autónoma Hui de Ningxia, en China, hemos considerado las relaciones dinámicas e interactivas entre los migrantes y el gobierno local, y hemos encontrado que el carácter participativo de las instituciones locales desempeña un papel fundamental para determinar el éxito o el fracaso de las políticas migratorias. Por tanto, los riesgos de empobrecimiento 
y de dislocación social no son inevitables consecuencias que se imponen a los migrantes: cuando éstos tienen oportunidad, son capaces de responder, adaptarse, influir y mejorar sus proyectos con el fin de promover sus intereses y mitigar los riesgos a los que están expuestos. 